\title{
Kinematic analysis of forelimb and hind limb joints in clinically healthy sheep
}

\author{
Luis G Faria', Sheila C Rahal ${ }^{1 *}$, Felipe S Agostinho', Bruno W Minto², Lídia M Matsubara', Washington T Kano', \\ Maira S Castilho ${ }^{1}$ and Luciane R Mesquita ${ }^{1}$
}

\begin{abstract}
Background: Variations associated with sex, age, velocity, breed and body geometry should be considered in the determination of kinematic parameters for a gait considered normal.

Therefore, this study aimed to evaluate kinematic patterns of forelimbs and hind limbs in clinically normal sheep from two different age groups walking at a constant velocity. The hypothesis was that the age may influence sagittal plane kinematic patterns. Fourteen clinically healthy female sheep were divided into Group 1 - seven animals aged from 8 to 12 months, and Group 2 - seven animals aged above 5 years. Before starting data collection, the sheep were trained to be conducted for walking in a pre-determined space at constant velocity. A minimum of 5 valid trials were obtained from the right and left sides of each sheep. Data were analyzed by use of a motion-analysis program. Flexion and extension joint angles (maximum, minimum, displacement), and angular velocity (maximum, minimum) were determined for the shoulder, elbow, carpal, hip, stifle, and tarsal joints.

Results: Within each group, no significant differences were observed between the right and left limbs in all kinematic variables. Significant differences were observed in the following kinematic parameters between $G 1$ and $G 2$ : minimum angle $(\mathrm{G} 1<\mathrm{G} 2)$, angular displacement $(\mathrm{G} 1>\mathrm{G} 2)$, maximum angular velocity $(\mathrm{G} 1>\mathrm{G} 2)$, minimum angular velocity ( $G 1>G 2)$ of the carpus; angular displacement $(G 1>G 2)$, minimum angular velocity $(G 1>G 2)$ of the shoulder; minimum angle $(G 1>G 2)$, angular displacement $(G 1<G 2)$ of the tarsus; maximum angular velocity $(G 1<G 2)$ of the stifle; maximum angular velocity $G 1>G 2$ of the hip. The lengths of both forelimbs and hind limbs differed between groups $(\mathrm{G} 1<\mathrm{G} 2)$. The Froude number differed between groups for forelimbs and hind limbs.
\end{abstract}

Conclusions: In conclusion, sheep of two different ages walking at a constant velocity present, within the same group, similar kinematic data between sides, and exhibit some differences in kinematic variables that may be age-related or body size. Further studies using sheep walking at similar Froude numbers are necessary to exclude the body size.

\section{Background}

Several instrumentation types are available for kinematic evaluation including films, video recordings, television/ computer, and optoelectronic systems that present considerable differences in terms of convenience and accuracy [1,2]. Most gait-analysis laboratories use a computer system to collect the data, in which markers placed at strategic locations on the body, or pre-determined anatomical landmarks, have the trajectories captured by specialized cameras $[2,3]$.

\footnotetext{
* Correspondence: sheilacr@fmvz.unesp.br

'Department of Veterinary Surgery and Anesthesiology, School of Veterinary Medicine and Animal Science - Univ Estadual Paulista (UNESP), Botucatu, SP, Brazil

Full list of author information is available at the end of the article
}

Kinematic gait analysis can be used to evaluate healthy individuals or individuals with diseases [1,4-7]. However, to enhance understanding of the abnormal gait requires determination of parameters for a gait considered normal; "normal" should be interpreted by taking into account variations associated with sex, age and body geometry $[1,8]$. In addition, morphological variations associated with the breeds should be considered in animals [3,6,9].

The stages of musculoskeletal growth and maturation of the central nervous system play an important role in gait analysis [1,10]; the walking pattern in an adult human is obtained at the age of 7 years, and gait parameter differences become stable at approximately 16 to 18 years old [11]. In addition, changes of locomotion may occur with advancing age. For example, elderly people may 
have altered excursion of joint movement, such as reduction in the total range of hip flexion and extension, in swing phase knee flexion, and in ankle plantar flexion [1].

Some kinematic studies have used the sheep as the experimental model [12-14]. Merino-mix sheep were used to evaluate the soft tissue coverage in the ascertainment of bone kinematics by means of skin-mounted markers [12]. Tridimensional stifle kinematics was applied to quantify in Suffolk-cross sheep the influence of the complete lateral meniscectomy [13]. Kinematic abnormalities measured by 3D stifle kinematic were correlated with degrees of early osteoarthritis in surgical models of anterior cruciate ligament/medial collateral ligament transection performed in Suffolk-cross sheep [14].

Therefore, the purpose of the present study was to evaluate kinematic patterns of forelimbs and hind limbs in clinically normal sheep from two different age groups walking at a constant velocity. The hypothesis was that age may influence sagittal plane kinematic patterns.

\section{Methods}

This study was approved by the Ethics Committee of School of Veterinary Medicine and Animal Science Univ Estadual Paulista (UNESP) (no. 42/2011-CEUA). Fourteen clinically healthy intact female sheep, client owned, all of the Santa Ines breed, were used: seven animals aged from 8 to 12 months and weighing 19-33 kg (G1), and seven animals aged more than 5 years and weighing 37-45 $\mathrm{kg}$ (G2). The owner of the sheep gave his consent to perform the experiment.

The animals were judged to be healthy on account of results of complete physical and orthopedic examinations. Before starting the kinematic analysis, the sheep were trained to be conducted for walking in a predetermined space at constant velocity by the same handler. Approximately seven days before the recordings, hoof trimming was accomplished.

\section{Data collection}

Kinematic analysis was performed using a 5-camera system (T10S camera - NIR 12.5; Vicon, Peak Performance Technologies Inc, Colorado, USA). For each analysis, the system was calibrated and a three-dimensional testing space ( $3 \mathrm{~m}$ in length $\times 2.5 \mathrm{~m}$ in width $\times 2 \mathrm{~m}$ in height) was established.

Each sheep was tagged with 11 retroreflective spherical markers $(1.8 \mathrm{~cm}$ in diameter) by a single investigator, as previously described [6]. Markers were placed on the skin using quick-drying glue over the dorsal point of the iliac crest, lateral prominence of the ischial tuberosity, greater trochanter of the femur, femorotibial joint between the lateral epicondyle of the femur and the fibular head, lateral malleolus, distal lateral aspect of metatarsi III and IV, the point of the cranial angle of the scapula, acromion of the scapulohumeral joint, lateral epicondyle of the humerus, styloid ulnar process, and distal lateral aspect of metacarpi III and IV.

A minimum of 5 valid trials were obtained and analyzed first from the left side, then the right side of each sheep; each trial included a complete stride cycle. Trials were considered valid if the animal walked within the predetermined velocity and acceleration, and without head movement or pulling on the halter. Data were analyzed by use of a motion-analysis program (Vicon Nexus). The velocity was maintained $1.1-1.3 \mathrm{~m} / \mathrm{s}$ and acceleration from -0.15 to $0.15 \mathrm{~m} / \mathrm{s}^{2}$ determined by a pressuresensitive walkway (Walkway High Resolution HRV4; Tekscan, South Boston, Massachusetts, USA).

The 11 individual markers were identified and labeled to construct a 3D stick-diagram representation of the sheep. The maximum, minimum and displacement values were obtained from normalized trials of each animal. A stride was defined from the beginning of the stance phase of one limb to the end of its swing phase. For the hind limb the beginning of the stance phase/swing phase was determined by the inversion moment of angular velocity of the hip joint at the end of each respective phase. For the forelimb, the beginning of stance phase/swing phase was determined by the inversion moment of angular velocity of the elbow joint at the end of each respective phase.

Flexion and extension joint angles (maximum, minimum, displacement), and angular velocity (maximum, minimum) were determined for the shoulder, elbow, carpal, hip, stifle, and tarsal joints. The length of each limb was established by the sum of the distances between each pair of markers on that limb.

The Froude number was calculated for both forelimbs and hind limbs as follow: $\mathrm{Fr}=\mathrm{v}^{2} / \mathrm{gl}(\mathrm{v}=$ velocity, $\mathrm{g}=\mathrm{ac}-$ celeration due gravity, $\mathrm{l}=$ limb length) [15].

\section{Statistical method}

To compare kinematic parameters between the right and left limbs within the same group, and data between the groups, one-way ANOVA was used followed by Tukey's post-hoc test. The values were expressed as mean \pm standard deviation, and the coefficients of variation (CV) were calculated. An independent sample $t$ test was used to compare lengths of the forelimbs and hind limbs and Froude number between groups. Differences were considered significant at $P<0.05$.

\section{Results}

Within each group, no significant differences were observed between the right and left limbs in all kinematic variables. Significant differences were observed in the following kinematic parameters between G1 and G2: minimum angle $(G 1<G 2)$, angular displacement $(G 1>G 2)$, 
maximum angular velocity (G1 $>G 2)$, minimum angular velocity $(\mathrm{G} 1>\mathrm{G} 2)$ of the carpus; angular displacement $(\mathrm{G} 1>\mathrm{G} 2)$, minimum angular velocity $(\mathrm{G} 1>\mathrm{G} 2)$ of the shoulder; minimum angle (G1 > G2), angular displacement $(\mathrm{G} 1<\mathrm{G} 2)$ of the tarsus; maximum angular velocity $(\mathrm{G} 1<\mathrm{G} 2)$ of the stifle; maximum angular velocity $\mathrm{G} 1>\mathrm{G} 2$ of the hip (Tables 1 and 2).

The lengths of both forelimbs $(P=0.008)$ and hind limbs $(P<0.001)$ differed between groups $(\mathrm{G} 1<\mathrm{G} 2)$. The differences were approximately $10 \mathrm{~cm}$ in forelimbs and $9 \mathrm{~cm}$ in hind limbs. The Froude number differed between groups $(P<0.001)$ for forelimbs $(\mathrm{G} 1=0.28 \pm 0.013$; $\mathrm{G} 2=0.24 \pm 0.016)$ and hind limbs (G1 $=0.27 \pm 0.012$; $\mathrm{G} 2=0.24 \pm 0.025)$.

\section{Discussion}

Normative studies have been carried out to characterize joint movement patterns in healthy dogs during trotting [6,16-22] or walking [23-25], which are considered symmetrical gaits due to the reciprocity of forelimb as well as hind limb movements [2,9]. In the present study each sheep's velocity was maintained from 1.1 to $1.3 \mathrm{~m} / \mathrm{s}$, which has been previously reported as walking locomotion [6,26].

Kinematic studies showed that to avoid interference, prior to data collection, some dog breeds require multiple training sessions [21] and others not [20]. In sheep studies it must also be considered that these animals become distressed due to changes in the social environment, especially when separated from the rest of the flock [27-29]. Thus, it is important that the sheep be halter-trained before obtaining kinematic data, as performed in the present study.

Although studies employing inverse dynamics have shown that the Labrador retriever dog breed may present a dominant side [30,31], sagittal kinematic studies in dogs did not detect significant differences between right and left sides in forelimbs or hind limbs suggesting symmetry $[6,19]$. Likewise, in the present study no statistical differences were observed between right and left limbs in all the kinematic parameters. In addition, studies using a pressuresensitive walkway found that kinetic and temporospatial parameters did not differ between sides [6,26].

In the forelimbs of both groups, the carpal joint had the highest angular displacement value, followed by the elbow joint, with the shoulder presenting the lowest value. In the hind limbs, the tarsal and stifle joints showed similar angular displacements, while the hip joint had the lowest value. Despite differing values, kinematic data from healthy dogs trotting on a treadmill showed similar patterns of sequence of angular displacement, for both forelimb and hind limbs $[7,19]$. A kinematic study that evaluated gait abilities of two lamb crossbreeds in three different environments also detected that the amplitude of angular variation rose gradually toward the distal extremity of the forelimbs and hind limbs [32]. On the other hand, a two-dimensional kinematic study in healthy dogs during walking found little variation in angular displacement values of the hind limb joints [24]. However, sheep perform stiff walk with hind limbs and compliant walk with forelimbs while

Table 1 Comparison of maximum angle $\left({ }^{\circ}\right)$, minimum angle $\left({ }^{\circ}\right)$, displacement angular velocity $\left({ }^{\circ}\right)$, maximum angular velocity $(\% / s)$, and minimum angular velocity $(\% / s)$ of the forelimb joints between Group 1 and Group 2

\begin{tabular}{|c|c|c|c|c|c|c|}
\hline \multirow[t]{2}{*}{ Variable } & & \multicolumn{2}{|l|}{ Group 1} & \multicolumn{2}{|l|}{ Group 2} & \multirow[b]{2}{*}{$P$ value } \\
\hline & & Mean \pm SD & CV & Mean \pm SD & CV & \\
\hline \multirow[t]{5}{*}{ Carpal } & Maximum angle & $176.03 \pm 3.20$ & 1.82 & $174.89 \pm 2.44$ & 1.40 & 0.296 \\
\hline & Minimum angle & $105.12 \pm 4.71$ & 4.48 & $116.52 \pm 10.82$ & 9.28 & 0.002 \\
\hline & Angular displacement & $70.91 \pm 3.58$ & 5.05 & $58.37 \pm 9.97$ & 17.08 & $<0.001$ \\
\hline & Maximum angular velocity & $794.57 \pm 110.34$ & 13.89 & $512.37 \pm 84.47$ & 16.49 & $<0.001$ \\
\hline & Minimum angular velocity & $-845.58 \pm 139.72$ & -16.52 & $-630.36 \pm 108.99$ & -17.29 & $<0.001$ \\
\hline \multirow[t]{5}{*}{ Elbow } & Maximum angle & $150.84 \pm 3.38$ & 2.24 & $149.97 \pm 4.58$ & 3.05 & 0.571 \\
\hline & Minimum angle & $106.93 \pm 5.13$ & 4.80 & $107.94 \pm 10.68$ & 9.90 & 0.755 \\
\hline & Angular displacement & $43.91 \pm 4.64$ & 10.56 & $42.04 \pm 8.73$ & 20.76 & 0.484 \\
\hline & Maximum angular velocity & $416.46 \pm 67.64$ & 16.24 & $401.42 \pm 73.60$ & 18.33 & 0.578 \\
\hline & Minimum angular velocity & $-363.90 \pm 60.62$ & -16.66 & $-331.36 \pm 54.89$ & -16.56 & 0.149 \\
\hline \multirow[t]{5}{*}{ Shoulder } & Maximum angle & $134.04 \pm 5.92$ & 4.42 & $131.60 \pm 6.68$ & 5.07 & 0.316 \\
\hline & Minimum angle & $121.62 \pm 5.20$ & 4.28 & $122.15 \pm 7.20$ & 5.89 & 0.826 \\
\hline & Angular displacement & $12.41 \pm 2.32$ & 18.72 & $9.45 \pm 2.19$ & 23.17 & 0.002 \\
\hline & Maximum angular velocity & $196.21 \pm 74.01$ & 37.72 & $147.64 \pm 48.38$ & 32.77 & 0.050 \\
\hline & Minimum angular velocity & $-197.97 \pm 73.54$ & -37.15 & $-110.87 \pm 23.07$ & -20.81 & 0.001 \\
\hline
\end{tabular}

$P$-values in bold represent significant differences $(P<0.05)$ between the mean of $G 1$ and $G 2$ variables. 
Table 2 Comparison of maximum angle $\left({ }^{\circ}\right)$, minimum angle $\left({ }^{\circ}\right)$, displacement angular velocity $\left({ }^{\circ}\right)$, maximum angular velocity $(\% / s)$, and minimum angular velocity $(\% / s)$ of the hind limb joints between Group 1 and Group 2

\begin{tabular}{|c|c|c|c|c|c|c|}
\hline \multirow[t]{2}{*}{ Variable } & & \multicolumn{2}{|l|}{ Group 1} & \multicolumn{2}{|l|}{ Group 2} & \multirow[b]{2}{*}{$P$ value } \\
\hline & & Mean \pm SD & CV & Mean \pm SD & CV & \\
\hline \multirow[t]{5}{*}{ Tarsal } & Maximum angle & $156.48 \pm 3.91$ & 2.50 & $154.59 \pm 5.13$ & 3.32 & 0.470 \\
\hline & Minimum angle & $126.44 \pm 5.54$ & 4.38 & $119.30 \pm 5.93$ & 4.97 & 0.003 \\
\hline & Angular displacement & $30.05 \pm 3.99$ & 13.27 & $35.30 \pm 4.45$ & 12.59 & 0.003 \\
\hline & Maximum angular velocity & $329.52 \pm 72.66$ & 22.05 & $360.03 \pm 58.18$ & 16.16 & 0.633 \\
\hline & Minimum angular velocity & $-336.94 \pm 61.62$ & -18.29 & $-340.06 \pm 54.45$ & -16.01 & 0.991 \\
\hline \multirow[t]{5}{*}{ Stifle } & Maximum angle & $156.48 \pm 6.95$ & 5.11 & $130.59 \pm 6.82$ & 5.22 & 0.084 \\
\hline & Minimum angle & $103.13 \pm 5.55$ & 5.38 & $95.60 \pm 8.48$ & 8.87 & 0.010 \\
\hline & Angular displacement & $32.85 \pm 4.73$ & 14.41 & $34.99 \pm 4.88$ & 13.95 & 0.426 \\
\hline & Maximum angular velocity & $313.96 \pm 53.66$ & 17.09 & $363.16 \pm 43.15$ & 11.88 & 0.026 \\
\hline & Minimum angular velocity & $-217.28 \pm 39.58$ & -18.22 & $-204.37 \pm 34.44$ & -16.85 & 0.590 \\
\hline \multirow[t]{5}{*}{ Hip } & Maximum angle & $156.48 \pm 9.01$ & 8.38 & $107.63 \pm 5.52$ & 5.12 & 0.999 \\
\hline & Minimum angle & $89.22 \pm 6.26$ & 7.02 & $91.81 \pm 5.32$ & 5.79 & 0.426 \\
\hline & Angular displacement & $18.30 \pm 7.32$ & 40.00 & $15.82 \pm 2.79$ & 17.65 & 0.399 \\
\hline & Maximum angular velocity & $213.79 \pm 139.77$ & 65.37 & $126.15 \pm 42.16$ & 33.42 & 0.031 \\
\hline & Minimum angular velocity & $-169.91 \pm-65.53$ & -38.57 & $-131.28 \pm 17.03$ & -12.98 & 0.050 \\
\hline
\end{tabular}

$P$-values in bold represent significant differences $(P<0.05)$ between the mean of $G 1$ and $G 2$ variables.

dogs present a stiff walk for both forelimbs and hind limbs [33].

With regard to angular velocity of the hind limbs, in both groups the tarsal joint produced the highest value, followed by the stifle joint, while the hip joint had the lowest value. In a study using measures of nonlinear dynamics in trotting dogs, it was observed that the hip joint also presented the lowest angular velocity but that the stifle joint showed the greatest value [17]. In the forelimbs of both groups, the carpal joint ranked highest, followed by the elbow joint, whereas the shoulder had the lowest value. Since the angular velocity corresponds to the rate of change in angular displacement with respect to elapsed time $[1,34,35]$, the data suggest that forces generated during locomotion in sheep require higher angular velocities for more distal joints related to increased angular displacement.

Humans may present a decrease in joint motion with advancing age [1] that has been associated with crosslinkage development between adjacent collagen fibrils and a decrease in the muscle mass, among others [36]. In the present study significant differences were observed in forelimbs in relation to angular displacement of the carpus $(\mathrm{G} 1>\mathrm{G} 2)$ associated with minimum angle $(\mathrm{G} 1<\mathrm{G} 2)$, and angular displacement $(\mathrm{G} 1>\mathrm{G} 2)$ of the shoulder. The data suggest that these joints display greater flexion-extension motion in younger sheep than in older ones probably due to the former's higher flexibility. In addition, higher angular velocity of the carpus was observed in G1 indicating that flexion and extension rates were faster in this group. Because this group had shorter forelimbs and hind limbs, and all animals walked at the same velocity, the data could suggest a compensation due to their different sizes [37]. The Froude number confirmed that limb length differences occurred between groups. However, to obtain the same Froude number the G1 animals would have to walk $0.1 \mathrm{~m} / \mathrm{s}$ more slowly than G2. To have this control over the velocity, the animal should be walking on a treadmill.

On the other hand, significant differences were observed in angular displacement of the tarsal joint $(\mathrm{G} 1<\mathrm{G} 2)$ associated with minimum angle (G1 $>$ G2). In a study to establish predictive performance values of trotting horses, despite the kinematic differences based on height differences, it was also reported that during growth, the joint angles become more extended [38], which may have influenced the necessity of lesser angular displacement with higher tarsal flexion in G1. Other alterations were lower maximum angular velocity of the stifle and higher maximum angular velocity of the hip in G1 compared to G2, indicating respectively slower and faster flexion of these joints in G1. However, skin-marker movement may produce errors in the calculations of joint angles [1,2,8,39] representing a limitation. This should be considered especially in areas with more pronounced soft tissue coverage such as the stifle and hip joints [12,39].

\section{Conclusions}

In conclusion, sheep of two different ages walking at a constant velocity present, within the same group, similar 
kinematic data between sides, and exhibit some differences in kinematic variables that may be age-related or body size. Further studies using sheep walking at similar Froude numbers are necessary to exclude the body size.

\section{Competing interests}

The authors have declared that no competing interests exist.

\section{Authors' contributions}

LGF, SCR, FSA and LRM conceived and designed the study; LMM, MSC and WTK helped collected the data, and BWM helped draft the manuscript; all authors read, contributed to and approved the final manuscript.

\section{Acknowledgements}

The authors are grateful to FAPESP (The State of São Paulo Research Foundation - 2012/02173-7) and CNPq (National Council for Scientific and Technological Development) for financial support.

\section{Author details}

'Department of Veterinary Surgery and Anesthesiology, School of Veterinary Medicine and Animal Science - Univ Estadual Paulista (UNESP), Botucatu, SP, Brazil. ${ }^{2}$ Department of Clinic and Veterinary Surgery, Faculdade de Ciências Agrárias e Veterinárias - UNESP, Jaboticabal, SP, Brazil.

\section{Received: 16 April 2014 Accepted: 5 December 2014}

Published online: 12 December 2014

\section{References}

1. Whittle MW: Gait Analysis: An Introduction. 4th edition. Edinburgh: Elsevier; 2007.

2. Gillette RL, Angle TC: Recent development in canine locomotor analysis. A review. Vet J 2008, 178:165-176.

3. Budsberg S: Canine kinetics and kinematics- uses and abuses. In Proceedings of the 14th European Society of Veterinary Orthopaedics and Traumatology Congress: 10-14 September 2008; Munich. Edited by Budsberg S. Germany: ESVOT; 2008:24-25

4. Marsolais GS, McLean S, Derrick T, Conzemius MG: Kinematic analysis of the hind limb during swimming and walking in healthy dogs and dogs with surgically corrected cranial ligament rupture. J Am Vet Med Assoc 2003, 222:739-743

5. Bockstahler BA, Henninger W, Muller M, Mayrhofer E, Peham C, Podbregar I: Influence of borderline hip dysplasia on joint kinematics of clinically sound Belgian Shepherd dogs. Am J Vet Res 2007, 68:271-276.

6. Agostinho FS, Rahal SC, Miqueleto NSML, Verdugo MR, Inamassu LR, El-Warrak AO: Kinematic analysis of Labrador Retrievers and Rottweilers trotting on a treadmill. Vet Comp Orthop Traumatol 2011, 24:185-191.

7. Miqueleto NSML, Rahal SC, Agostinho FS, Siqueira EGM, Araújo FAP, El-Warrak AO: Kinematic analysis in healthy and hip-dysplastic German Shepherd dogs. Vet J 2013, 195:210-215.

8. Baker R: Gait analysis methods in rehabilitation. J NeuroEng Rehab 2006, 3:1-10.

9. DeCamp CE: Kinetic and kinematic gait analysis and the assessment of lameness in the dog. Vet Clin North Am Small Anim Pract 1997, 27:825-840.

10. Howland DR, Bregman BS, Goldberger ME: The development of quadrupedal locomotion in the kitten. Exp Neurol 1995, 135:93-107.

11. Pasparakis D, Darras N: Normal walking: Principles, basic concepts, terminology 3-dimensional clinical gait analysis. EEXOT 2009, 60:183-194.

12. Taylor WR, Ehrig RM, Duda GN, Schell H, Seebeck P, Heller MO: On the influence of soft tissue coverage in the determination of bone kinematics using skin markers. J Orthop Res 2005, 23:726-734.

13. Beveridge JE, Shrive NG, Frank CB: Meniscectomy causes significant in vivo kinematic changes and mechanically induced focal chondral lesions in a sheep model. J Orthop Res 2011, 29:1397-1405.

14. Frank CB, Beveridge JE, Huebner KD, Heard BJ, Tapper JE, O'Brien JOE, Shrive NG: Complete $A C L / M C L$ deficiency induces variable degrees of instability in sheep with specific kinematic abnormalities correlating with degrees of early osteoarthritis. J Orthop Res 2012, 30:384-392.

15. Alexander RMN: The gaits of bipedal and quadrupedal animals. Int $J$ Robot Res 1984, 3:49-59.
16. DeCamp CE, Soutas-Little RW, Hauptman J, Olivier B, Braden T, Walton A: Kinematic gait analysis of the trot in healthy Greyhounds. Am J Vet Res 1993, 54:627-634.

17. Marghitu DB, Kincaid SA, Rumph PF: Nonlinear dynamics stability measurements of locomotion in healthy Greyhounds. Am J Vet Res 1996 57:1529-1535

18. Schaefer SL, DeCamp CE, Hauptman JG, Walton A: Kinematic gait analysis of hind limb symmetry in dogs at the trot. Am J Vet Res 1998, 59:680-685.

19. Gillette $R L$, Zebas $C J$ : A two-dimensional analysis of limb symmetry in the trot of Labrador retrievers. J Am Anim Hosp Assoc 1999, 35:515-520.

20. Owen MR, Richards J, Clements DN, Drew ST, Bennett D, Carmichael S: Kinematics of the elbow and stifle joints in Greyhounds during treadmill trotting - an investigation of familiarisation. Vet Comp Orthop Traumatol 2004, 17:141-145.

21. Clements DN, Owen MR, Carmichael S, Reid SWJ: Kinematic analysis of the gait of 10 Labrador retrievers during treadmill locomotion. Vet Rec 2005, 156:478-481.

22. Colborne GR, Walker AM, Tattersall AJ, Fuller CJ: Effect of trotting velocity on work patterns of the hind limbs of Greyhounds. Am J Vet Res 2006, 67:1293-1298.

23. Hottinger HA, Decamp CE, Olivier BN, Hauptman JG, Soutas-Little RW: Noninvasive kinematic analysis of the walk in healthy large-breed dogs. Am J Vet Res 1996, 57:381-388.

24. Feeney LC, Lin CF, Marcellin-little DJ, Tate AR, Queen RM, YU B: Validation of two-dimensional kinematic analysis of walk and sit-to-stand motions in dogs. Am J Vet Res 2007, 68:277-282.

25. Kim J, Rietdyk S, Breur GJ: Comparison of two-dimensional and threedimensional systems for kinematic analysis of the sagittal motion of canine hind limbs during walking. Am J Vet Res 2008, 69:1116-1122.

26. Kim J, Breur GJ: Temporospatial and kinetic characteristics of sheep walking on a pressure sensing walkway. Can Vet J 2008, 72:50-55.

27. Parrott RF, Houpt KA, Misson BH: Modification of the responses of sheep to isolation stress by the use of mirror panels. An Behav Sci 1988, 19:331-338

28. Bouissou MF, Porter RH, Boyle L, Ferreira G: Influence of a conspecific image of own vs. different breed on fear reactions of ewes. Behav Proc 1996, 38:37-44.

29. Hargreaves AL, Hutson GD: Handling systems for sheep. Livest Product SCI 1997, 49:121-138.

30. Colborne GR: Are sound dogs mechanically symmetric at trot? No, actually. Vet Comp Orthop Traumatol 2008, 21:294-301.

31. Colborne GR, Good L, Cozens LE, Kirk LS: Symmetry of hind limb mechanics in orthopedically normal trotting Labrador Retrievers. Am J Vet Res 2011, 72:336-344.

32. Abourachid A, Laville E: Kinematic study of the locomotion of two crossbreds of lambs. Ann Zootec 1997, 46:219-230.

33. Jayes AS, Alexander RMN: Mechanics of locomotion of dogs (Canis familiaris) and sheep (Ovis aries). J Zool London 1978, 185:289-308.

34. Robertson DGE, Caldwell GE: Planar kinematics. In Research Methods in Biomechanics. Edited by Robertson DGE, Caldwell GE, Hamill J, Kamen G, Whittlesey SN. Champaign: Human kinetics; 2004

35. Weigel JP, Arnold G, Hicks DA, Millis DL: Biomechanics of rehabilitation. Vet Clin North Am: Sm Anim Pract 2005, 35:1255-1285.

36. Shephard RJ: Aging and exercise. In Encyclopedia of sports medicine and science. Edited by Fahey TD. Internet Society for Sport Science; 1998. [http://www.sportsci.org/encyc/agingex/agingex.html]

37. Alexander RMN, Jayes AS: A dynamic similarity hypothesis for the gaits of quadrupedal mammals. J Zool London 1983, 201:135-152.

38. Back W, Schamhardt HC, Hartman W, Bruin G, Barneveld A: Predictive value of foal kinematics for the locomotor performance of adult horses. Res Vet Sci 1995, 59:64-69.

39. Kim SY, Kim JY, Hayashi K, Kapatkin AS: Skin movement during the kinematic analysis of the canine pelvic limb. Vet Comp Orthop Traumatol 2011, 24:326-332.

doi:10.1186/s12917-014-0294-4

Cite this article as: Faria et al:: Kinematic analysis of forelimb and hind limb joints in clinically healthy sheep. BMC Veterinary Research 2014 10:294 